\title{
Phylogenetic community ecology as an approach for studying old ideas on competition in the plankton: opportunities and challenges
}

\author{
Steven M. VAMOSI* \\ Department of Biological Sciences, 2500 University Drive NW, University of Calgary, Calgary AB T2N 1N4, Canada \\ *Corresponding author: smvamosi@ucalgary.ca
}

\begin{abstract}
There is a long history in ecology of studying the mechanisms that may promote the long-term co-occurrence of phytoplankton, and zooplankton to a lesser degree, in aquatic systems. The recent emergence of using phylogenetic information to understand community assembly may represent a new tool for tackling old questions about competition in plankton, although studies using such methods in this way are rare to date. Here, I provide an overview of the general methods developed in related studies, briefly review existing works, and discuss various conceptual and methodological challenges that researchers should be aware of before applying these methods to plankton communities.
\end{abstract}

Key words: coexistence, competition, habitat filtering, niche differences, phylogenetic community structure, plankton.

Received: June 2013. Accepted: August 2013

\section{INTRODUCTION}

A longstanding conundrum in community ecology is the frequent observation of long-term co-occurrence of species occupying the same trophic level, especially in relatively simple environments. Hutchinson (1961) is famous for introducing the so-called paradox of the plankton, noting according to the principle of competitive exclusion (Hardin, 1960) ... we should expect that one species alone would outcompete all the others so that in a final equilibrium situation the assemblage would reduce to a population of a single species (Hutchinson 1961, pg. 137). Others (Cropp and Norbury, 2012) quote the same principle of exclusion (Gause, 1934; Hardin, 1960) to indicate that the number of species cannot exceed the number of limiting resources; in the case of phytoplankton, this might increase the null expectation roughly by an order of magnitude (e.g., 15-20 species based on Hutchinson, 1961; Wilson, 1990). Regardless of which value is used, deviations from these expectations in real communities can be quite striking, as evidenced by the species lists for 33 well-studied lakes (see Tab. 1 of Dodson et al., 2000). Phytoplankton species richness, for example, ranged from 20 species to a staggering 234 species (mean=114). Species richness of zooplankton (cladocerans and copepods), which constitutes a second example of the paradox of the plankton (Hutchinson, 1961; Fox et al., 2010; Masclaux et al., 2012), also deviated from the former expectation, albeit less markedly so (mean=13, range 3-25). Many potential mechanisms have been advanced for the coexistence of so many species, including niche differences, trade-offs between competitive ability and predation risk and the storage effect; I will not go into any detail on them, but these and other mechanisms have been amply reviewed elsewhere (Wilson, 1990; Roy and Chattopadhyay, 2007; Fox et al., 2010; Hayashi, 2011).

A closely allied area of investigation in evolutionary ecology has focused on mechanisms that may promote the coexistence of closely related species, such as congeners. Because of the considerable evolutionary history shared among congeners, it is expected that they will tend to resemble each other more than distantly related species and, furthermore, that they may compete most strongly for limiting resources (Darwin, 1859). Again making reference to the principle of exclusion, one would predict that the probability of long-term co-occurrence would accordingly be negatively correlated with phylogenetic relatedness. However, congeners are frequently observed to co-occur in local phytoplankton (and other) communities. In Lake Biwa, for example, it is estimated that there are 88 phytoplankton species (Horie, 1984), representing five Classes and 52 genera. Several genera were represented by three or more species, with the desmid Staurastrum being represented by seven species. With regard to zooplankton, this pattern was nonexistent for copepods, very weak for cladocerans and extremely pronounced for rotifers, with 80 species of the latter group being represented by only 32 genera and three genera (Lecane, Brachionus, Trichocerca) contributing roughly a third of the total diversity (10, 9, and 8 species, respectively). Niche differences and character displacement are often cited as facilitating the co-occurrence of congeneric animal taxa 
(Schluter, 2000; Lu et al., 2011). For primary producers, these explanations are complicated by arguments that competition for nutritionally essential (or, non-substitutable) resources such as nitrogen or phosphorus may favour character convergence rather than character divergence (Abrams, 1987, 2012; Fox and Vasseur, 2008). Depending on how frequently such convergence occurs between distantly related species, this may complicate the interpretation of phylogenetic signal calculated for relevant traits or the degree to which phylogenetic relatedness predicts the outcome of species interactions. Indeed, a meta-analysis by Cahill et al. (2008) did not find a significant relationship between strength of interspecific competition and phylogenetic relatedness across a sample of 142 species of eudicot and monocot plants. Interestingly, the relationship was significant within one group (monocots) but not the other.

Here, I discuss the field of phylogenetic community ecology, with a special emphasis on how it may be applied to plankton communities. Broadly speaking, phylogenetic community ecology marries community ecology approaches with phylogenetic information to inform studies of community organization (Webb et al., 2002). The seminal papers in this area are generally considered to be Webb (2000) and Webb et al. (2002), with the former paper introducing the modern approach to measuring phylogenetic community structure and the latter providing a broader overview of three major classes of questions that can be addressed when combining information on phylogenetic relatedness of species with distributional and trait data for species. Described by some as a bandwagon (Fox, 2012), the field of phylogenetic ecology has been witness to a veritable explosion of papers in the past decade or so, with the majority of them focusing on plant communities (Vamosi et al., 2009). Turning our attention to plankton, literature searches reveal ongoing interest in community structure and common application of molecular techniques. However, searching the ISI Web of Science $S c i$ ence Citation Index Expanded database with search criteria TS=phylogenetic community AND phytoplankton or TS=phylogenetic community AND zooplankton led to only one paper from the latter search (Helmus et al., 2010). Changing the second term to the less specific *plankton led to three additional hits, but all focused on bacterioplankton (Fernandez-Guerra and Casamayor, 2012). Although not exhaustive, with a relevant paper by Litchman et al. (2010) not being uncovered by these searches, similar searches with other taxa typically lead to many more hits (S.M. Vamosi, personal observation). In the main text, I will cover three areas: i) an overview of historical and modern approaches to incorporating information about species relatedness into studies of community organization, ii) a consideration of the findings of a few attempts to apply a phylogenetic community ecol- ogy to plankton communities, and iii) general and specific concerns that researchers intending to apply these approaches to plankton communities should be aware of. Finally, I end with a brief synopsis and look ahead to future investigations in this area.

\section{PHYLOGENETIC COMMUNITY ECOLOGY - A BRIEF REVIEW}

The factors influencing the presence (absence) and relative abundance of a set of potentially interacting species in a sample of communities have long been the subject of study in ecology. An early series of investigations, related to the focal topic of the current paper, applied so-called 'species-to-genus ratios' in biogeographical studies (Elton, 1946; see Järvinen, 1982 for a review). In the absence of good information on hypothesized phylogenetic relationships among all coexisting species, researchers would calculate the average number of species per genus for focal communities and attempted to correlate variance in these ratios with attributes of the communities, such as distance to mainland. A low species to genus ratio was taken to imply the coexistence of distantly related species, whereas a high species to genus ratio was interpreted as evidence for the converse. Furthermore, a finding of low species to genus ratios was taken as evidence that closely related species (e.g., congeners) competed strongly, which was limiting their ability to coexist (Elton, 1946). Elton (1946), for example, included a few studies of plankton, which generally revealed low ratios (e.g., 1.19-1.32 for zooplankton from three lakes, pointing to the possible influence of competition in determining local community composition. Although it was applied in a large number of studies - indeed, literature searches reveal limited uses even in the present era (Drovetski et al., 2010) - a number of concerns have been raised over the years about its use. A major issue sprung from the fact that, unless all genera are monotypic, the number of genera will always be less than that of the number of species. Consequently, larger samples will produce higher species to genus ratios than small samples (Gotelli and Colwell, 2001), severely limiting the ability to make meaningful comparisons among communities with markedly different species richness values, such as those on islands versus those on mainlands.

The modern field of phylogenetic community ecology was formally launched by Webb (Webb, 2000; Webb et al., 2002; see also reviews by Cavender-Bares et al., 2009; Kembel, 2009; Vamosi et al., 2009). Webb (2000) capitalized on the rapid accumulation of molecular phylogenies (APG, 1998; see Webb, 2000 for others) and combined that with data on the composition of tree species in 28 0.16-ha plots in Gunung Palung National Park, Indonesia. His method involved generating a hypothesized phylogeny for all 324 species encountered across all plots (i.e., the regional pool), and comparing the 
phylogenies of species in plots with randomly generated phylogenies of equal species richness. Doing so represents a large leap forward from species to genus ratios because the entire phylogenetic structure of each community is considered, each genus is not counted as a single entity, and disputes about generic assignments become largely irrelevant. Two metrics were introduced in this paper: Net Relatedness Index (NRI) and Nearest Taxa Index (NTI; see Vamosi et al. 2009 for a summary of several alternative metrics). NRI provides a measure of mean pairwise nodal distance, or the phylogenetic clumpedness of taxa over the whole pool phylogeny (Webb, 2000, pg. 147), whereas NTI is a measure of the mean nearest nodal distance, or the degree of clumping among terminal taxa (or, tips) in the community. He found that NRI did not differ significantly from the random expectation, whereas NTI was significantly greater than expected, indicating greater clumping near the tips than expected by chance, which is now typically referred to as phylogenetic clustering. These findings were interpreted as support for the hypothesis that diversity in the forest was maintained by habitat partitioning, although it would more commonly be called habitat filtering now i.e., different habitats will select for groups of species that possess traits that allow them to successfully persist there. Provided that there is phylogenetic signal for the traits (i.e., phylogenetic distance between species is positively associated with similarity of their trait values), it is expected that different habitats will be characterized by different groups of closely related species (Fine and Kembel, 2011).

\section{PROGRESS TO DATE}

As alluded to previously, there appears to be relatively limited application of the phylogenetic ecology approaches outlined here to studying phytoplankton or zooplankton communities. Interestingly, plants are the dominant taxon (and, accordingly, primary producers are the dominant trophic level) in studies of terrestrial communities. Helmus et al. (2010) tested the hypothesis that disturbance should result in increased phylogenetic clustering using a rich dataset that contained zooplankton records from 18 disturbed and 16 control lakes. This investigation is notable for setting up a priori hypotheses about patterns expected in the two groups, rather than simply fitting post-hoc explanations to the results emerging from the analyses. A broad range of physical, chemical and biological disturbances were included in the study, although increases/decreases in water levels, increases in nutrient levels, acidification and increases/decreases addition/removal of fish species were common themes. For their regional pool, they obtained sequences for 56 of 67 taxa from Genbank and reconstructed hypothesized phylogenetic relationships to produce three most-parsimonious trees, after which the 11 taxa without sequence data were grafted onto the trees. Cobbling together supertrees for the regional pool using a combination of previously collected sequences, sequences generated specifically for the study and/or manually editing the phylogeny's Newick string to insert unsequenced taxa with reference to taxonomic affiliations is perhaps unsurprisingly common in phylogenetic community ecology studies (see Vamosi and Vamosi, 2007). Even when the results are argued to be robust to the underlying regional pool phylogeny, I believe the field would benefit from i) more researchers producing robust, dated phylogenies for their species with multiple markers and ii) further investigations into the scenarios under which results are most sensitive to phylogenetic quality. Helmus et al. (2010) calculated the phylogenetic species variability (PSV) of each zooplankton sample; this metric ranges from 0 (indicating clustering) to 1 (indicating evenness) and is most analogous to NRI (Helmus et al., 2007). As predicted, the phylogenetic clustering of zooplankton communities increased on average after disturbances were applied to lakes. The mean effect $(-2.3 \%$ for 16 disturbance-reference paired comparisons, $-2.7 \%$ based on unpaired difference between 18 disturbed and 16 control lakes) was quite modest, with the change in PSV ranging from +0.02 to -0.04 in 14 lakes and reaching -0.06 to -0.12 in only four lakes. Regardless, the approaches taken in this investigation can be viewed as an excellent starting point for related studies on phytoplankton community assembly.

\section{PHYLOGENETIC COMMUNITY ANALYSIS WORKFLOW}

Because my literature searches uncovered relatively few phylogenetic ecology studies focused on plankton communities, I have sketched out a rudimentary workflow (Fig. 1; see also Swenson 2013 for an independently derived analogue). Three caveats are probably best spelled out here. First, I strongly caution the reader not to treat this as a foolproof or exhaustive recipe for successfully carrying out future studies, just as phylogenetic clustering or evenness should not immediately be equated with evidence for habitat filtering or competitive exclusion, respectively (see also next section). That being said, it is also worth noting that few existing studies have considered/met all points shown, especially the final one (i.e., validating the results of one investigation by analyzing another set of communities or by carrying out manipulative experiments). Finally, this is almost certainly a biased view of how to proceed, and many readers will recognize the resemblance to the model-selection philosophy espoused by Burnham and Anderson (2002). With those reservations (and the concerns that follow below) in mind, it is my hope that this workflow provides some guidance to those contemplating adopting these approaches. 


\section{GENERAL CONCERNS}

Increasingly, concerns have been expressed about various elements of the phylogenetic community ecology enterprise. The issue that will likely prove most difficult to resolve was that raised by Mayfield and Levine (2010), which is that competitive exclusion can lead to either phylogenetic clustering or evenness. Readers are encouraged to carefully read the original paper, which bases its arguments on modern coexistence theory (see also Chesson, 2000; Adler et al., 2007), but I will attempt to sketch out the main points here. In essence, it is argued that the potential for coexistence of competing species is mediated by the interaction between two types of differences, niche differences and competitive ability differences (see Fig. 2 of

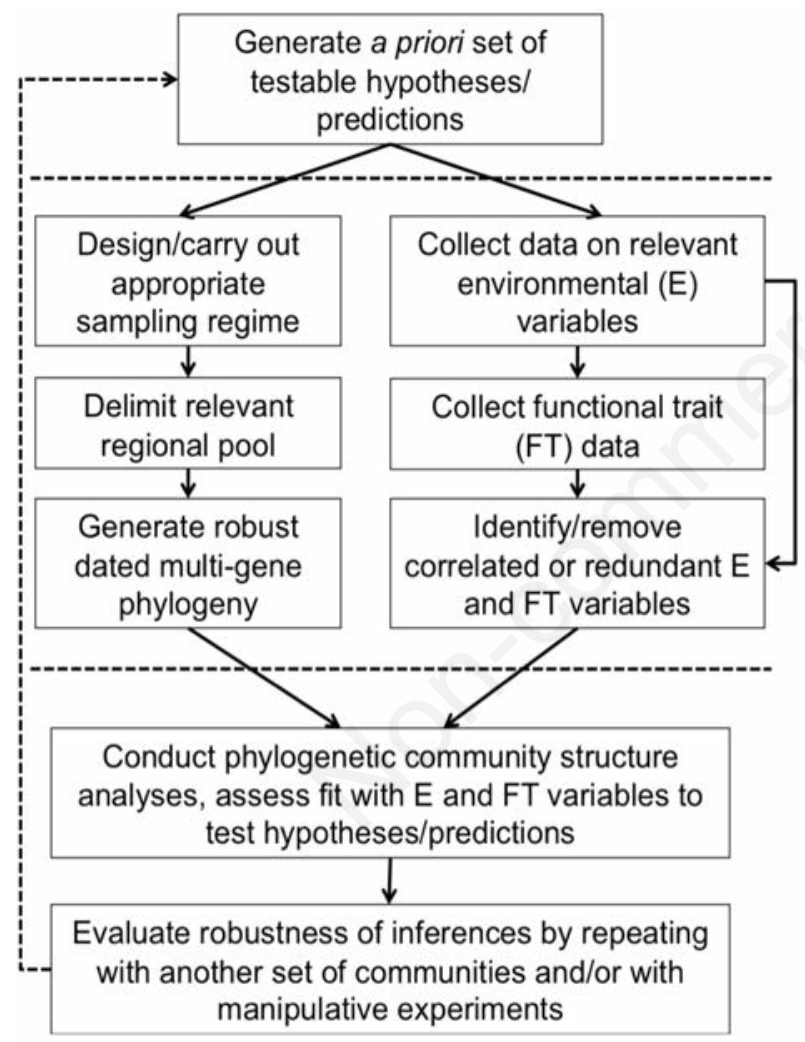

Fig. 1. A potential workflow for a phylogenetic community structure analysis, illustrating the basics of the main steps, such as properly motivating the study with a priori hypotheses and predictions (box in top section), obtaining relevant pieces of data (boxes in middle section), and carrying out and validating analyses (boxes in bottom section). Arrows should not be interpreted too strictly; minimally, collecting functional trait data need not wait on environmental data. Calculating phylogenetic signal for traits has not been explicitly included in the diagram, but would likely be undertaken either in tandem with generating robust phylogenies or as part of the phylogenetic community structure analyses.
Mayfield and Levine). On their own, niche differences positively affect the likelihood of coexistence whereas the converse is true for differences in competitive ability. Getting back to the issue, the two outcomes in phylogenetic structure are possible when there is a phylogenetic signal to niche differences (potentially leading to evenness) and competitive ability differences (leading to clustering). Whether these arguments will prove fatal to studies of phylogenetic community structure or instead will stimulate the introduction of a revised framework remains to be seen. At present it minimally means that researchers should include ecological relevant traits and have a good understanding of how they may affect coexistence, because it is not sufficient to demonstrate phylogenetic conservatism in easily measurable traits, which may not actually be functional traits (Vamosi et al., 2009; Swenson 2013).

Although generally supportive of studies of phylogenetic community structure, Vamosi et al. (2009) encountered several shortcomings in studies that were available at that time. The one that I would like to highlight here is one that is increasingly being discussed in the literature, which is increased transparency of raw data upon which subsequent inferences are based. In collating studies for what would be the first quantitative survey of such studies, we encountered several papers that did not report such basic information as number of sites included and total number of species. As endorsed by a growing number of leading journals (e.g., Molecular Ecology, Evolution), uploading raw data (e.g., accession numbers for DNA sequences, Nexus files of the phylogenies for the regional pool and local communities, ecological trait data, species lists for all sites) to permanent archives such as Dryad (http://datadryad.org/), ideally starting with the review process, would be desirable for all future studies.

A final comment is that calculating NRI and NTI values (or similar; see Vamosi et al., 2009 for a treatment of other metrics) for a series of communities for which data were handy should not be viewed as an end result (see also Fig. 1). Certainly, many older papers on plankton community structure present data on species distributions, which could be revisited with information on phylogenetic relatedness of the constituent species and data on traits; from this perspective, two classic papers on zooplankton and phytoplankton community structure look very promising (Lynch, 1979; Lynch and Shapiro, 1981). Independent of criticisms such as those raised by Mayfield and Levine (2010), causation cannot be inferred from pattern alone, yet it is not uncommon to find papers on phylogenetic community structure that do little more than speculate about mechanisms from a series of NRI/NTI values. Webb (2000; pg. 146), in launching the field, was pragmatic about the place of such metrics in the bigger picture: Fully addressing the relationship between the phylogenetic relatedness of a community and the ecological similarity of its species will be a 
large, complicated research program, requiring the explicit measurement of ecological traits for individual species. Without the full study of the autecology of individual species, interpreting phylogenetic structure in terms of ecological similarity must rely on the (justified) expectation of the conservatism of autecology during lineage evolution and, thus, must be made tentatively. Admittedly, my own investigations into the phylogenetic community ecology of aquatic communities are best viewed as representing only the early stages of a proper research program (Vamosi and Vamosi, 2007; Silver et al., 2012).

\section{SPECIFIC CONCERNS}

With regard to plankton, especially phytoplankton, I expect researchers will grapple the most with two issues: i) reconstructing hypothesized phylogenetic relationships, and ii) determining and measuring the relevant (suites of) functional traits. The former is actually a multi-faceted issue, resulting from the high species richness of phytoplankton, which is a somewhat artificial grouping of several speciesrich taxa, some of which are very distantly related to the others (e.g., Cyanophyceae vs all others, Chlorophyceae vs Dinophyceae) and the difficulties in delineating appropriate community scales and regional pools. For studies focusing on marine communities, the regional pool for any study will be some hard-to-define subset of a total pool of 3000-5000 species (Tett and Barton, 1995). Although one can simply equate the regional pool as simply being the total list of species from all local sites, this could unintentionally result in not observing particular plankton taxa (e.g., entire genera) in local sites even though they are present in the larger area. Such a scenario might apply, for example, when sampling a relatively homogenous set of lakes (e.g., all with relatively low $\mathrm{pH}$ ), none of which contain groups of species found in the region but sensitive to those particular conditions (Holopainen, 1992). Because these taxa would not be included in a regional pool that was simply the sum of species encountered in sampled lakes, this $\mathrm{pH}$-mediated habitat filtering would not being detected in this case (see also Webb, 2000; Fine and Kembel, 2011). With regard to freshwater communities, although the local regional pool is likely to be smaller there is still a fair amount of uncertainty in the phylogenetic relationships within and among the major subgroups (Bruggeman, 2011; Friedl and Rybalka, 2012), and researchers will still need to select their local sampling sites and characterize their regional pools with care. The latter issue is partially also the result of high species richness, with detailed ecological data likely missing from a large proportion of extant phytoplankton species (Bruggeman, 2011). Phyto-PhyloPars (Bruggeman et al., 2009; Bruggeman, 2011; http://www.ibi.vu.nl/programs/phylopars/phytoplankton) is a promising approach to dealing with these data gaps, using a phylogenetic approach to estimate data on several traits, namely four size measurements (length, diameter, sur- face area, volume), maximum growth rate, phosphate affinity and edibility, extrapolated from over 1000 observations on freshwater species. These are unlikely to be the only relevant traits, but it is encouraging to note that others have identified possible candidates and advocated the creation of an easily accessible electronic trait database for phytoplankton (Litchman et al., 2010). Such efforts could be very fruitful if paired with existing taxonomic databases, such as AlgaeBase (Guiry and Guiry, 2013; http://www.algaebase. org/). I would argue that neither component (i.e., phylogenetic relatedness and functional traits) should be viewed as more important than the other. Indeed, Swenson (2013) has enumerated the advantages and disadvantages of both types of data/analyses and I believe that they are most informative when considered together.

\section{CONCLUSIONS}

Over fifty years ago, Hutchinson (1961) introduced his famous paradox of the plankton, spurring considerable research into the mechanisms that promote the long-term co-occurrence of many species in relatively simple environments. Argued by some to no longer be a paradox (Cropp and Norbury, 2012), the maintenance of diversity at local, regional and global scales nevertheless remains a fundamentally important question in ecology and evolutionary biology. With reference to phytoplankton communities, a survey of the literature reveals continued interest into these questions from pure and applied perspectives (Beaver et al., 2013; Chust et al., 2013; Larroude et al., 2013). In this paper, I provided a brief overview of the growing field of phylogenetic community ecology in the hopes of encouraging more researchers to apply this framework to outstanding questions in phytoplankton (and zooplankton) community assembly. The study of responses of phytoplankton communities to disturbances by Helmus et al. (2010) provides a good example of this approach, which I recommend that others consult as they plan and carry out related investigations. Hopefully, the schematic I provided in Fig. 1 will help guide the initial explorations of some future practitioners. Once more studies have documented prevailing trends in phylogenetic community structure, it is hoped that these approaches can be applied to other questions, such as the impacts of phylogenetic diversity on ecosystem function (Cadotte et al., 2011) and stability (Cadotte et al., 2012). Although there are outstanding issues that need to be resolved, investigating the phylogenetic structure of plankton communities may enhance ongoing investigations and, in some cases, reveal fruitful areas for future work.

\section{ACKNOWLEDGMENTS}

I thank Diego Fontaneto and Angela Boggero for the opportunity to contribute to this special issue, three 
anonymous reviewers for thoughtful comments on an earlier version of the manuscript, and the Natural Sciences and Engineering Research Council of Canada (NSERC) Discovery Grants program for supporting my research.

\section{REFERENCES}

Abrams PA, 1987. Alternative models of character displacement and niche shift. I. Adaptive shifts in resource use when there is competition for nutritionally nonsubstitutable resources. Evolution 41:651-661.

Abrams PA, 2012. The eco-evolutionary responses of a generalist consumer to resource competition. Evolution 66:3130-3143.

Adler PB, HilleRisLambers J, Levine JM, 2007. A niche for neutrality. Ecol. Lett. 10:95-104.

Beaver JR, Casamatta DA, East TL, Havens KE, Rodusky AJ, James RT, Tausz CE, Buccier KM, 2013. Extreme weather events influence the phytoplankton community structure in a large lowland subtropical lake (Lake Okeechobee, Florida, USA). Hydrobiologia 709:213-226.

Bruggeman J, 2011. A phylogenetic approach to the estimation of phytoplankton traits. J. Phycol. 47:52-65.

Bruggeman J, Heringa J, Brandt BW, 2009. PhyloPars: estimation of missing parameter values using phylogeny. Nucleic Acids Res. 37:W179-W184.

Burnham KR, Anderson DR, 2002. Model selection and multimodel inference: a practical information-theoretic approach, $2^{\text {nd }}$ edition. Springer, New York: $488 \mathrm{pp}$.

Cadotte MW, Carscadden K, Mirotchnick N, 2011. Beyond species: functional diversity and the maintenance of ecological processes and services. J. Appl. Ecol. 48:1079-1087.

Cadotte MW, Dinnage R, Tilman D, 2012. Phylogenetic diversity promotes ecosystem stability. Ecology 93: S223-S233.

Cahill JF Jr, Kembel SW, Lamb EG, Keddy PA, 2008. Does phylogenetic relatedness influence the strength of competition among vascular plants? Perspect. Plant Ecol. Evol. Syst. 10:41-50.

Cavender-Bares J, Kozak KH, Fine PVA, Kembel SW, 2009. The merging of community ecology and phylogenetic biology. Ecol. Lett. 12:693-715.

Chesson P, 2000. Mechanisms of maintenance of species diversity. Annu. Rev. Ecol. Syst. 31:343-366.

Chust G, Irigoien X, Chave J, Harris RP, 2013. Latitudinal phytoplankton distribution and the neutral theory of biodiversity. Global Ecol. Biogeogr. 22:531-543.

Cropp R, Norbury J, 2012. The mechanisms of coexistence and competitive exclusion in complex plankton ecosystem models. Ecosystems 15:200-212.

Darwin C, 1859. On the origin of species by means of natural selection, or the preservation of favoured races in the struggle for life. John Murray, London: 502 pp.

Dodson SI, Arnott SE, Cottingham KL, 2000. The relationship in lake communities between primary productivity and species richness. Ecology 81:2662-2679.

Drovetski SV, Zink RM, Ericson PGP, Fadeev IV, 2010. A multilocus study of pine grosbeak phylogeography supports the pattern of greater intercontinental divergence in Holarctic boreal forest birds than in birds inhabiting other high-latitude habitats. J. Biogeogr. 37:696-706.

Elton C, 1946. Competition and the structure of ecological communities. J. Anim. Ecol. 15:54-68.
Fernandez-Guerra A, Casamayor EO, 2012. Habitat-associated phylogenetic community patterns of microbial ammonia oxidizers. PLoS ONE 7:e47330.

Fine PVA, Kembel SW, 2011. Phylogenetic community structure and phylogenetic turnover across space and edaphic gradients in western Amazonian tree communities. Ecography 34:552-565.

Fox JW, 2012, October 9. Can the phylogenetic community ecology bandwagon be stopped or steered? A case study of contrarian ecology. [Web log comment] Retrieved from http://dynamicecology.wordpress.com/2012/10/09/can-thephylogenetic-community-ecology-bandwagon-be-stoppedor-steered-a-case-study-of-contrarian-ecology/

Fox JW, Vasseur DA, 2008. Character convergence under competition for nutritionally essential resources. Am. Nat. 172:667-680.

Fox JW, Nelson WA, McCauley E, 2010. Coexistence mechanisms and the paradox of the plankton: quantifying selection from noisy data. Ecology 91:1774-1786.

Friedl T, Rybalka N, 2012. Systematics of the green algae: a brief introduction to the current status, p. 259-280. In: U. Lüttge, W. Beyschlag, B. Büdel and D. Francis (eds.), Progress in Botany 73, Springer Berlin Heidelberg.

Gause GF, 1934. The struggle for existence. Williams and Wilkins, Baltimore: 163 pp.

Gotelli NJ, Colwell RK, 2001. Quantifying biodiversity: procedures and pitfalls in the measurement and comparison of species richness. Ecol. Lett. 4:379-391.

Guiry MD, Guiry GM, 2013. AlgaeBase. World-wide electronic publication, National University of Ireland, Galway. http://www.algaebase.org; accessed on 15 May 2013.

Hardin G, 1960. The competitive exclusion principle. Science 131:1292-1298.

Hayashi T, 2011. Monospecific planktonic diatom assemblages in the Paleo-Kathmandu lake during the middle Brunhes Chron: implications for the paradox of the plankton. Palaeogeogr. Palaeoclimatol. Palaeoecol. 300:46-58.

Helmus MR, Bland TJ, Williams CK, Ives AR, 2007. Phylogenetic measures of biodiversity. Am. Nat. 169: E68-E83.

Helmus MR, Keller W, Paterson MJ, Yan ND, Cannon CH, Rusak JA, 2010. Communities contain closely related species during ecosystem disturbance. Ecol. Lett. 13:162-174.

Holopainen IJ, 1992. The effects of low pH on planktonic communities. Case history of a small forest pond in eastern Finland. Ann. Zool. Fennici 28:95-103.

Horie S, editor, 1984. Lake Biwa. Monographiae Biologicae 54. Dr. W. Junk Publishers, Dordrecht: 654 pp.

Hutchinson GE, 1961. The paradox of the plankton. Am. Nat. 95:137-145.

Järvinen O, 1982. Species-to-genus ratios in biogeography: a historical note. J. Biogeogr. 9:363-370.

Kembel SW, 2009. Disentangling niche and neutral influences on community assembly: assessing the performance of community phylogenetic structure tests. Ecol. Lett. 12:949-960.

Larroude S, Massei N, Reyes-Marchant P, Delattre C, Humbert JF, 2013. Dramatic changes in a phytoplankton community in response to local and global pressures: a 24-year survey of the River Loire (France). Glob. Change Biol. 19:1620-1631.

Litchman E, de Tezanos Pinto P, Klausmeier CA, Thomas MK, Yoshiyama K, 2010. Linking traits to species diversity and 
community structure in phytoplankton. Hydrobiologia 653:15-28.

Lu X, Gong G, Ma X, 2011. Niche segregation between two alpine rosefinches: to coexist in extreme environments. Evol. Biol. 38:79-87.

Lynch M, 1979. Predation, competition, and zooplankton community structure: an experimental study. Limnol. Oceanogr. 24:253-272.

Lynch M, Shapiro J, 1981. Predation, enrichment, and phytoplankton community structure. Limnol. Oceanogr. 26:86-102.

Masclaux H, Bec A, Bourdier G, 2012. Trophic partitioning among three littoral microcrustaceans: relative importance of periphyton as food resource. J. Limnol. 71:261-266.

Mayfield MM, Levine JM, 2010. Opposing effects of competitive exclusion on the phylogenetic structure of communities. Ecol. Lett. 13:1085-1093.

Roy S, Chattopadhyay J, 2007. Towards a resolution of 'the paradox of the plankton': a brief overview of the proposed mechanisms. Ecol. Complex. 4:26-33.

Schluter D, 2000. The ecology of adaptive radiation. Oxford University Press, Oxford: 296 pp.
Silver CA, Vamosi SM, Bayley SE, 2012. Temporary and permanent wetland macroinvertebrate communities: phylogenetic structure through time. Acta Oecol. 39: 1-10.

Tett P, Barton ED, 1995. Why are there about 5000 species of phytoplankton in the sea? J. Plankton Res. 17:1693-1704.

Vamosi JC, Vamosi SM, 2007. Body size, rarity, and phylogenetic community structure: insights from diving beetle assemblages of Alberta. Divers. Distrib. 13:1-10.

Vamosi SM, Heard SB, Vamosi JC, Webb CO, 2009. Emerging patterns in the comparative analysis of phylogenetic community structure. Mol. Ecol. 18:572-592.

Webb CO, 2000. Exploring the phylogenetic structure of ecological communities: an example for rain forest trees. Am. Nat. 156:145-155.

Webb CO, Ackerly DD, McPeek MA, Donoghue MJ, 2002. Phylogenies and community ecology. Annu. Rev. Ecol. Syst. 33:475-505.

Wilson JB, 1990. Mechanisms of species coexistence: twelve explanations for Hutchinson's 'paradox of the plankton': evidence from New Zealand plant communities. New Zeal. J. Ecol. 13:17-42. 